\title{
From the Editor's Desk: The Clustering of America, and the Conundrum of Caring for the Complex Adult
}

\author{
Malathi Srinivasan, $M D^{7}$ and Mitchell D. Feldman, MD, MPhi ${ }^{2}$ \\ 'Department of Medicine, Division of General Internal Medicine, University of California, Sacramento, CA, USA; ${ }^{2}$ Department of Medicine, \\ Division of General Internal Medicine, University of California, San Francisco, San Francisco, CA, USA.
}

J Gen Intern Med 26(5):459

DOI: $10.1007 / \mathrm{s} 11606-011-1684-\mathrm{y}$

() Society of General Internal Medicine 2011

I n his book "The Clustered World," Michael J. Weiss describes how socio-demographically, the American population can be seen as 62 clusters, defined by specific characteristics across geographic regions. Weiss notes increasing fragmentation at all levels of society, as our insular communities live separate, nonoverlapping lives. Whether Blue Blood Estates, Gray Power, Young Influentials, Hard Scrabble, or Mines and Mills, our lives intersect regularly only in public venues or in health care.

This issue of JGIM highlights the health challenges faced by these insular, vulnerable clusters of American society-and the need for more evidence to guide their care. One of our most vulnerable clusters is the poor elderly, whose complex medical disorders are heightened by lack of physiologic and financial reserve. Zulman and colleagues examine the lack of evidence to guide appropriate medical care in the elderly. In their systematic review, they examine all randomized therapeutic trials published in 2007 in five major medical journals assessing morbidity and mortality outcomes. Of the 109 studies reviewed, $20 \%$ of randomized clinical trials (RCTs) excluded elderly patients at the outset, almost half excluded patients based on criteria which would effectively exclude the elderly, and only a quarter included quality of life or health status outcomes important to elderly patients. These exclusions are understandable, as RCTs focus on answering a specific question, and prefer homogeneity to complexity.

Can observational studies of natural cohorts provide the evidence needed to improve vulnerable patient care? Perhaps.

Published online March 26, 2011
In this issue, Shrank explores the biases and limitations of major observational studies, including biases implicit in sampling healthy adult users of and adherers to medical care. While methodologically more challenging than enrolling and following nurses (e.g. the Nurses Health Study) or health conscious individuals using supplements, sampling complex older patients for both RCTs and observational studies may help us utilize health care resources more appropriately.

As we contemplate providing better care to the complex older adult, Lindquist explores the ability of paid non-family caregivers to provide appropriate care to their elderly charges. In this study, one- third of caregivers had poor health literacy, while 60\% made errors with pillbox text medication. (See editorial by Sudore and Covinsky in this issue).

General internists are well aware that lack of time, resources and evidence can serve as barriers to providing the best care for our complex elderly, many of whom have often outlived their cohorts. Whether caring for a 78-year-old woman with advanced COPD, CHF, and OA whose family has died, or a 92-year-old active man with cognitive decline and a severe anxiety disorder, we struggle with important questions about their care. How do we reach across our American clusters to gather the resources to help them improve (or maintain) their health? How much benefit will accrue to our patients, if we prescribe one course of action in comparison with anothernot just for longevity, but for quality of life? We need more evidence and better system-wide approaches to provide comprehensive care to our complicated, vulnerable elderly, clustered in sight but often out of reach.

Corresponding Author: Malathi Srinivasan, MD; Department of Medicine, Division of General Internal Medicine, University of California, Davis; 4150 V. Street, Suite 2400, Sacramento, CA 95817, USA (e-mail: malathi@ucdavis.edu). 